\title{
Evolution of Operating System
}

\author{
Vivek Laxkar ${ }^{1}$, Pandurang Lahane ${ }^{2}$, Nikita Abhang ${ }^{3}$, Mohanish Shinde ${ }^{4}$, HOD Mithun Mhatre ${ }^{5}$ \\ Students, Bharati Vidyapeeth's Institute of Technology, Kharghar, India ${ }^{1,2,3,4}$ \\ HOD, Computer Technology, Bharati Vidyapeeth's Institute of Technology, Kharghar, India ${ }^{5}$
}

\begin{abstract}
An operating system is an interface between the hardware and user. It has simplified the usage of machine in every technological aspect. In this the evolution of the operating system has been explained such as Batch Processing System, Multiprogramming System, Multitasking System, Time Sharing System and Desktop System. In this we have also explained the detailed working of each and every type of Operating system over the period of time. In this we have also listed and explained the type of view i.e. User view and System view.
\end{abstract}

Keywords: Operating system, Mobile operating system, base of system translator, user view, system view

\section{INTRODUCTION}

An operating system act as a system translator between the user of a computer and computer hardware. An operating system is also known as a resource manager which manages the various software as well as hardware resources. The primary goal of operating system is to provide convenience for the user and efficient operation of the computer system. An operating system interacts with the hardware of the computer to perform the different tasks. Operating system is also called as platform for the machine. Operating system falls in the category of system software, Many resources such as input and output devices, memory files storage, CPU etc. In simple word operating system is the interface between the user and machine. Therefore operating system is the resource manager that act as a host.

\section{LITERATURE REVIEW}

Embedded systems have become ubiquitous and are found in numerous application domains such as sensor networks, medical devices, and smart appliances. ${ }^{[1]}$ Software flaws in such systems can range from minor nuisances to critical security failures and malfunctions. ${ }^{[2]}$ COMMOS is an operating system Architecture developed to support shared persistent objects in distributed systems. This paper describes its support for flexible conference. ${ }^{[3]}$ This paper summarizes a process of operating system adaption to an Intel Atom processor. The main process of an operating system is a more complicated processor family, without destroying the original modules of system or charging their functionality. ${ }^{[4]}$

\section{METHODOLOGY}

1. Batch processing system

- In earlier punch cards were used to record the user's jobs.

- To avoid the continuous human operators devotion the concept of batch processing grown up

- A batch stands for the sequence of user jobs. In this type of operating system the job sequence was decided by an operator.

- The jobs were recognized with the special markers which were indicating the start and the end of those jobs.

- It can use spooling technique.

2.Multiprogramming system

- The process of executing multiple number of programs simultaneously or concurrently is known as multiprogramming.

- Multiprogramming is the most important feature of an operating system.

- When this current job waits operating system switches to another job and so on this way multi-way programming.

- The other alternatives for keeping CPU and other devices continuously in use.

- Multiprogramming is more useful as compared to Spooling and Buffering.

3. Multitasking system

- A multitasking operating system is having unique feature of supporting multiple process execution at a time.

- Many jobs can be handled in multitasking system.

- Multiple programs can run simultaneously.

- It increases the speed of execution by doing resource management. 
Vol. 7, Issue 8, August 2018

- The example of multitasking operating system is UNIX, Linux and windows 2000.

4.Time sharing system

- The operating system that allows a number of different user to share the single computer system for a variety of application at the same time is known as time sharing system.

- A time sharing system is basically a multiprogramming, multitasking and multi-user environment of a large computer system.

- Time sharing allows to many users to share computer simultaneously.

- Time shared systems are more difficult and expensive to build.

- The old example of time sharing operating system is “The Dartmouth Time Sharing operating System".

5.Desktop system

- Desktop system are generally a single user system. i.e. it is dedicated to a single user. Desktop system are also called as personal computers.

- The desktop system is user friendly system.

- CPU utilization is not important.

- User convenience and responsiveness.

- Desktops can run several different types of operating system. e.g. Windows, MAC.OS/2, UNIX, Linux etc.

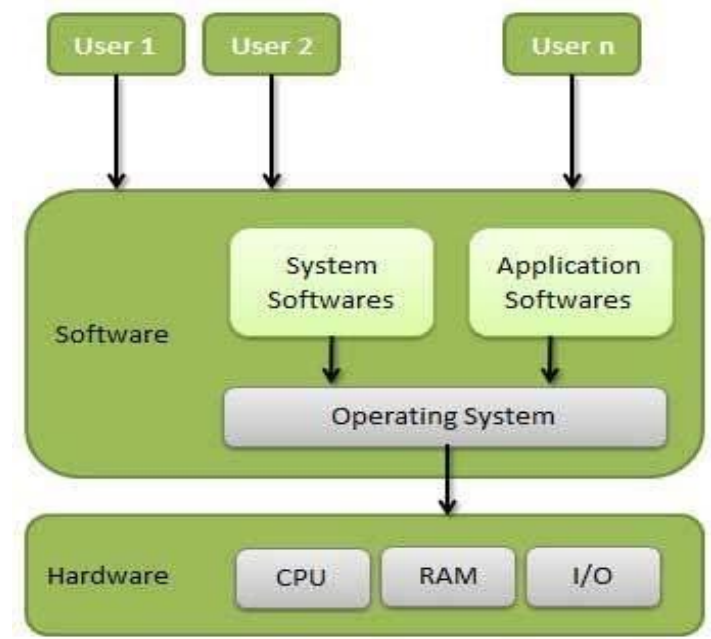

Two views of operating system

1) User view: The user view of the computer refers to the system being used. Such system is designed for one user to monopolize its resources, to maximize the work. The software means set of program clubbed together to achieve a particular set of task or activities and user contains various embedded system and home application in user view system.

2) System view: System view can record data from the embedded target system while it is running. System view can in parallel to debugger and do continuous recording. The recorded data is analyzed and the system behavior is visualizing in different views. System view consist of various applications of system view as well as interface and it also contain system software and operating system.

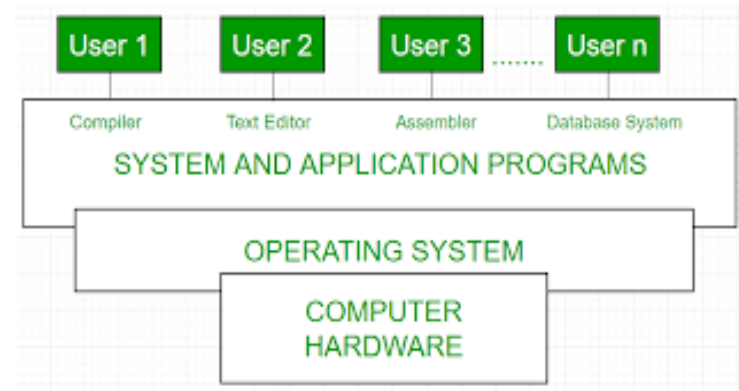

Latest version of operating system: The Latest version of operating system is windows 10 pro. Windows 10 pro is now replacing windows 10 , in addition to all of home edition's features, privacy tools such as Domain Join, Group Policy Management, Bit locker, Enterprise Mode Internet Explorer (EMIE), Assigned Access 8.1, Remote Desktop, Client Hyper-V, and Direct Access. 
Features of Windows 10 Pro

- Assigned access enables you to create a lockdown environment where uses interact with only one app when they signed into a specified account.

- In assigned access, users won't be able to get to the desktop, Start menu, and any other app, making it easier to safeguard your personal file.

\section{Bit Locker}

- Bit Locker is a kind of encryption software which allows you fully secure your device from potential hackers.

- Microsoft has made some improvement to this service in windows 10 Pro.

- $\quad$ The updated Bit Locker allows you to encrypt individual files and keep them alongside unencrypted ones.

- Microsoft Hyper-V, Formerly Known as Windows Server Virtualization, is a native hypervisor.

- Virtualizing is another benefit of Windows 10 Pro, although few need to use it.

- You'll also need to have a CPU which support virtualization.

4) Domain Join

- Windows 10 Pro supports joining a domain, allowing PCs to be added to a corporate network.

- In Windows 10 Home, you can't do this unless you use a Microsoft account rather than a local user account.

- This means the laptop or PC you want to use at a work and access files on servers need to be running Windows 10 Pro.

Trusted Boot

- Trusted Boot(tboot) is an open source pre-kernel/VMM module that user Intel(R) Trusted Execution Technology (Intel(R) TXT) to perform a measured and verified launch of an Operating System kernel/VMM.

- Secure Boot is for the boot loader and Trusted Boot is for drivers/software after Secure Boot is done.

\section{FUTURE SCOPE}

Operating system plays an important role it works like a coordinator or mediator between the computer hardware and user. Since it is system software, it can interact with hardware. User uses different program or application called as software which is a most important job done by operating system. Therefore the operating system contains various application and different types of latest version such as Ubuntu, Macintosh, Marcos x, Microsoft windows 10, Linux, Android and many more operating are being developed. The operating system is going to simplify the life of the human. Its' user interface not only simplify the interaction between the user and the machine but it is also very responsive which is going to be 4 times more than in present in the future.

\section{CONCLUSION}

Without operating system the computer cannot run the application and we cannot do work on the computer. Therefore operating system is very important in computer. It manages computer hardware resources and provides common services for computer program. It is a group of computer hardware and computer software like kernel, turbo c, java, etc. In this we have also explained the evolution of the operating system and how it has evolved over the years.

\section{REFERENCES}

[1]. Yanking Li, M. Potkonjak and W. Wolf "Real-time operating systems for embedded computing" https://ieeexplore.ieee.org/document/628899/

[2]. P. Pradhan and T.-C. Chiueh "Operating systems support for programmable cluster-based Internet routers" https://ieeexplore.ieee.org/document $/ 798381 /$

[3]. Feng Huang "Operating system support for flexible coherence in distributed object systems" https://ieeexplore.ieee.org/document/470560/

[4]. Ladislav Kobza, Martin Vojtko and Tibor Krajcovic "Migration of a Modular Operating System to a Intel Atom Processor" https://ieeexplore.ieee.org/document/7275242/

[5]. B. Gowing and V. Cahill "Making meta-object protocols practical for operating systems" https://ieeexplore.ieee.org/document/470579/

[6]. A.A. Hanish "Operating systems and communication protocols" https://ieeexplore.ieee.org/document/470561/

[7]. M. Balasubramanian and N. S. Usha "Studies on open source real time operating systems: For vehicle suspension control" https://ieeexplore. ieee.org/document/8070787/

[8]. Emil Delic, Karolin Löser and Ali Hayek "Platform independent safety-critical operating system" https://ieeexplore.ieee.org/document/ $7222952 /$

[9]. Jimin Kim and Minsoo Ryu "APRIX: A Master-Slave Operating System Architecture for Multiprocessor Embedded Systems"

[10]. T.E. Anderson "The case for application-specific operating systems" https://ieeexplore.iee.org/document/275682/ 